\title{
MiR-23a regulates TGF- $\beta$-induced epithelial-mesenchymal transition by targeting E-cadherin in lung cancer cells
}

\author{
MENGRU CAO $^{1,2}$, MASAHIRO SEIKE ${ }^{1}$, CHIE SOENO $^{1}$, HIDEAKI MIZUTANI ${ }^{1}$, KAZUHIRO KITAMURA ${ }^{1}$, \\ YUJI MINEGISHI $^{1}$, RINTARO NORO $^{1}$, AKINOBU YOSHIMURA $^{1}$, LI CAI $^{2}$ and AKIHIKO GEMMA ${ }^{1}$ \\ ${ }^{1}$ Department of Internal Medicine, Division of Pulmonary Medicine/Infection and Oncology, \\ Nippon Medical School, Tokyo 113-8603, Japan; ${ }^{2}$ Department of Medical Oncology, \\ The Third Affiliated Hospital of Harbin Medical University, Harbin, P.R. China
}

Received March 30, 2012; Accepted June 1, 2012

DOI: $10.3892 /$ ijo.2012.1535

\begin{abstract}
Transforming growth factor- $\beta$ (TGF- $\beta$ )-induced epithelial-mesenchymal transition (EMT) has been shown to be related to the pathogenesis of various diseases including lung cancer. Recently, microRNAs (miRNA) have been recognized as a new class of genes involved in human tumorigenesis. MiR-23a/24/27a is a miRNA cluster located in chromosome 19 p13.12, which can function as an oncogene in several human cancers. In this study, we analyzed miR-23a/24/27a expression in 10 non-small cell cancer (NSCLC) cell lines by real-time PCR analysis. Correlation between expression of these miRNAs and TGF- $\beta /$ Smad signaling was evaluated. We found that miR-23a could be regulated by TGF- $\beta 1$ in a Smad-dependent manner in A549 lung adenocarcinoma cells showing the EMT phenomenon. Knockdown of miR-23a partially restored E-cadherin expression under conditions of TGF- $\beta 1$ stimulation. In contrast, overexpression of miR-23a could suppress E-cadherin expression and stimulate EMT. Furthermore, A549 cells with overexpressed miR-23a were more resistant to gefitinib compared to the parental cells. These findings suggest that miR-23a regulates TGF- $\beta$-induced EMT by targeting E-cadherin in lung cancer cells and may be useful as a new therapeutic target in NSCLC.
\end{abstract}

\section{Introduction}

Lung cancer continues to be a leading cause of cancer death both in Japan and worldwide (1) and, despite recent improvements in chemotherapies and molecular-targeted therapies, the prognosis remains poor (2-5). Patient selection based on a specific biomarker is one strategy that could lead to improved

Correspondence to: Dr Masahiro Seike, Department of Internal Medicine, Division of Pulmonary Medicine/Infection and Oncology, Nippon Medical School, 1-1-5, Sendagi, Bunkyo-ku, Tokyo 113-8603, Japan

E-mail: mseike@nms.ac.jp

Key words: microRNA, epithelial-mesenchymal transition, transforming growth factor- $\beta$, Smad, lung cancer lung cancer treatments. Although some biomarkers predictive of metastasis, prognosis and drug sensitivity have already been reported in lung cancer, more sensitive and specific biomarkers could facilitate the development of novel therapeutic applications (6-8).

Epithelial-mesenchymal transition (EMT) comprises a complex series of reversible events that can lead to the loss of epithelial cell adhesion and the induction of a mesenchymal phenotype (9). Thus, EMT is characterized by the loss of epithelial differentiation markers including E-cadherin and the induction of mesenchymal markers such as vimentin and fibronectin. EMT can be induced by transforming growth factor- $\beta 1$ (TGF- $\beta 1$ ) (10). The Smad pathway is a major transducer of TGF- $\beta$ signaling (11). Smad 2 and Smad3 are phosphorylated by the TGF- $\beta$ type I receptor and form complexes with Smad4 (11). These complexes accumulate in the nucleus of the cell, regulating the transcription of target genes and playing critical roles in the control of cell proliferation, differentiation, apoptosis and cell migration. In response to TGF- $\beta$, the TGF- $\beta$ receptors also activate alternative signaling effectors, such as mitogen-activated protein kinase, phosphatidylinositol-3 kinase, and Rho-like GTPases (11). It has been recognized that EMT plays a pivotal role in several diverse processes during embryonic development, chronic inflammation and fibrosis (12). Recently, several studies demonstrated that EMT was correlated with carcinogenesis, metastasis and poor prognosis in various human cancers, including those of the lung (13-16). Furthermore, EMT has been reported to be related to reduced sensitivity and acquired resistance to epidermal growth factor tyrosine kinase inhibitors (EGFR-TKI) in lung cancer cells (17-19). Taken together, these findings demonstrate that the suppression of EMT could be used as a potential target for treatment of lung cancer.

MicroRNA (miRNAs) are a class of short single-stranded noncoding endogenous RNAs, approximately 18-24 nucleotides in length, which post-transcriptionally modulate gene expression by either inhibiting translation or inducing mRNA degradation (20). MiRNAs have been recognized as a new class of genes involved in human tumorigenesis $(21,22)$ and recently they have been shown to be diagnostic, prognostic and therapeutic biomarkers in lung cancer (22-25). For example, high miR-155 expression and low let-7a expression, as independent risk 
factors, have a negative prognostic impact on outcome in lung adenocarcinoma patients (23). The miR-17-92 cluster functions as an oncogene, and has been shown to promote lung cancer carcinogenesis (24). We previously reported that the inhibition of miR-21, whose upregulation is associated with EGFR mutations, can be a therapeutic strategy, either as a monotherapy or in combination with EGFR-TKI treatment (25). These findings suggest that miRNA can serve as a novel therapeutic target as well as diagnostic and prognostic marker in lung cancer.

A recent study reported that a specific cluster of miRNA, miR-23a/24/27a, was induced by TGF- $\beta$ in a Smad-dependent manner in hepatocellular carcinoma (HCC) cells (26). Upregulation of these miRNAs were able to suppress TGF- $\beta$ induced growth suppressive activities in HCC cells. In this present study, we analyzed miR-23a/24/27a expression in nonsmall cell cancer (NSCLC) cells and evaluated the correlation between its expression and TGF- $\beta / \mathrm{Smad}$ signaling. We found that miR-23a could be induced by TGF- $\beta$ in a Smad-dependent manner in A549 cells. In addition, overexpression of mature miR-23a reduced E-cadherin expression and stimulate the EMT phenomenon which is involved in tumorigenesis. Furthermore, in these A549 cells, inhibition of miR-23a could also partially suppress TGF- $\beta$-induced EMT, while overexpression was associated with the tendency for EGFR-TKI resistance. We have demonstrated that, in lung cancer cells, miR-23a is regulated by the TGF- $\beta /$ Smad pathway and plays a critical role in EMT through the targeting of E-cadherin.

\section{Materials and methods}

Cell culture. We used 10 NSCLC cell lines: A549, PC9, PC14, LC2/ad, RERF-LC-KJ, RERF-LC-OK adenocarcinoma (AC) cell lines and PC1, PC10, LK2 and SQ5 squamous cell carcinoma cell lines (SCC) for this study. An immortalized tracheal cell line (BET2A) was used as a normal control cells. A549 and BET2A were purchased from the American Type Culture Collection (ATCC, Manassas, VA, USA); RERF-LC-KJ, RERF-LC-OK, LC2-ad, LC2/Ad and SQ5 were obtained from the RIKEN Cell Bank (Ibaraki, Japan); and PC1, PC9, PC10 and PC14 were obtained from Immuno-Biological laboratories (Gunma, Japan). NSCLC cell lines were maintained in RPMI-1640 medium (Gibco, Carlsbad, CA, USA) supplemented with $10 \%$ fetal bovine serum (FBS). BET2A was maintained in RPMI-1640 medium with 5\% FBS.

RNA extraction and real-time quantitative reverse transcription-PCR. Total RNA was extracted from the BET2A and NSCLC cell lines with Trizol reagent (Invitrogen, Carlsbad, CA, USA). The miR-23a, miR-24 and miR-27a expression levels were quantified by quantitative reverse transcriptionPCR (qRT-PCR) using TaqMan ${ }^{\circledR}$ MicroRNA Assay System (Applied Biosystems, Foster City, CA). RNU66 (PN 4373382) was used as an internal control (Applied Biosystems). MiRNA expression was quantified and reported as $2^{-\Delta \Delta C t}$ value (27).

Antibodies and western blot analysis. Cells were lysed in buffer containing $50 \mathrm{mM}$ Tris- $\mathrm{HCl}$, pH 7.6, $150 \mathrm{mM} \mathrm{NaCl}$, $0.1 \%$ sodium dodecyl sulfate, $1 \%$ Nonidet P-40, and $0.5 \%$ sodium-deoxycholate. The lysates were kept on ice for $30 \mathrm{~min}$, and then centrifuged at $13000 \mathrm{x} \mathrm{g}$ for $30 \mathrm{~min}$. The super- natant was collected and then $10 \mu \mathrm{g}$ of each of the proteins was separated by SDS-PAGE on $10 \%$ gels and transferred to nitrocellulose membrane. After being blocked in $5 \%$ skimmed milk, the membrane was incubated with $\operatorname{Smad} 2 / 3, \beta$-actin (Cell Signaling Technology, Beverley, MA, USA), E-cadherin, $\mathrm{N}$-cadherin and vimentin (Santa Cruz Biotechnology, Santa Cruz, CA, USA) antibodies. Proteins were detected by immunoblotting using ECL-Plus reagents (GE Healthcare Bio-Science Corp, Piscataway, NJ, USA).

Oligonucleotide transfection. TGF- $\beta 1$ was purchased from R\&D system (Minneapolis, MN, USA). Cells were treated with $5 \mathrm{ng} / \mathrm{ml}$ TGF- $\beta 1$ for the indicated time. Small interference RNA (siRNA) targeting Smad2/3 was purchased from Dharmacon Research Inc. (Lafayette, CO, USA) and the homologous negative control was obtained from Invitrogen. MiR-23a inhibitor, its negative control, miR-23a precursor (Pre-miR-23a) and its cognate negative control (Pre-miR-ctl) were synthesized by Ambion (Ambion). Pre-miR-23a and miR-23a inhibitor were transfected using Lipofectamine ${ }^{\mathrm{TM}} 2000$ reagent $24 \mathrm{~h}$ after seeding, as per the manufacturer's instructions (Invitrogen). Transfections of precursor and inhibitor complexes were added to cells at a final concentration of $40 \mathrm{nM}$. Six hours after the transfection was performed, the transfection medium was replaced, and after $24 \mathrm{~h}, 5 \mathrm{ng} / \mathrm{ml}$ TGF- $\beta 1$ was added to the medium which was then incubated at $37^{\circ} \mathrm{C}$ for $48 \mathrm{~h}$.

Growth inhibition assay. Gefitinib was purchased from Seleck Chemicals (Houston, TX, USA). A549 cells (5,000 cells/well) were seeded into 96-well plates for $24 \mathrm{~h}$. After being treated with Pre-miR-ctl or Pre-miR-23a, at a final concentration of $40 \mathrm{nM}$ for $24 \mathrm{~h}$, the cells were incubated in the various concentrations of gefitinib for $72 \mathrm{~h}$ at $37^{\circ} \mathrm{C}$. Then, MTS was added to each well and the cells were incubated for a further $2 \mathrm{~h}$ at $37^{\circ} \mathrm{C}$, after which absorbance was measured using a microplate reader with a test wavelength of $450 \mathrm{~nm}$. The $\mathrm{IC}_{50}$ value was defined as the concentration needed for $50 \%$ reduction of the growth by treatment with gefitinib.

\section{Results}

MiR-23a, miR-24 and miR-27a expression in lung cancer cells. We first investigated miR-23a, miR-24 and miR-27a expression levels in NSCLC cell lines, including $6 \mathrm{AC}$ cell lines and 4 SCC cell lines. Expression levels of these mature miRNAs were examined by qRT-PCR (Fig. 1). Although these miRNAs belonged to the same miRNA cluster, their expression levels varied among the 10 cell lines.

MiR-23a expression is directly induced by TGF- $\beta 1$ in a Smaddependent manner. A recent study reported that $\mathrm{miR}-23 \mathrm{a} / 24 / 27 \mathrm{a}$ was induced by TGF- $\beta$ in a Smad-dependent manner in HCC cells (26). Smad pathway is known as a major transducer of TGF- $\beta$ signaling (11). We evaluated the correlation between miR-23a, miR-24 and miR-27a expression levels and Smad expression. High expression of miR-23a was observed in those NSCLC cells which also overexpressed Smad2/3. Thus, 5 cell lines (A549, LC-2/ad, ABC1, PC1 and SQ5) showed high expression of miR-23a and Smad2/3 (Fig. 2A), while low expression of miR-23a and Smad2/3 was found in the other 5 cell lines (PC9, 
A
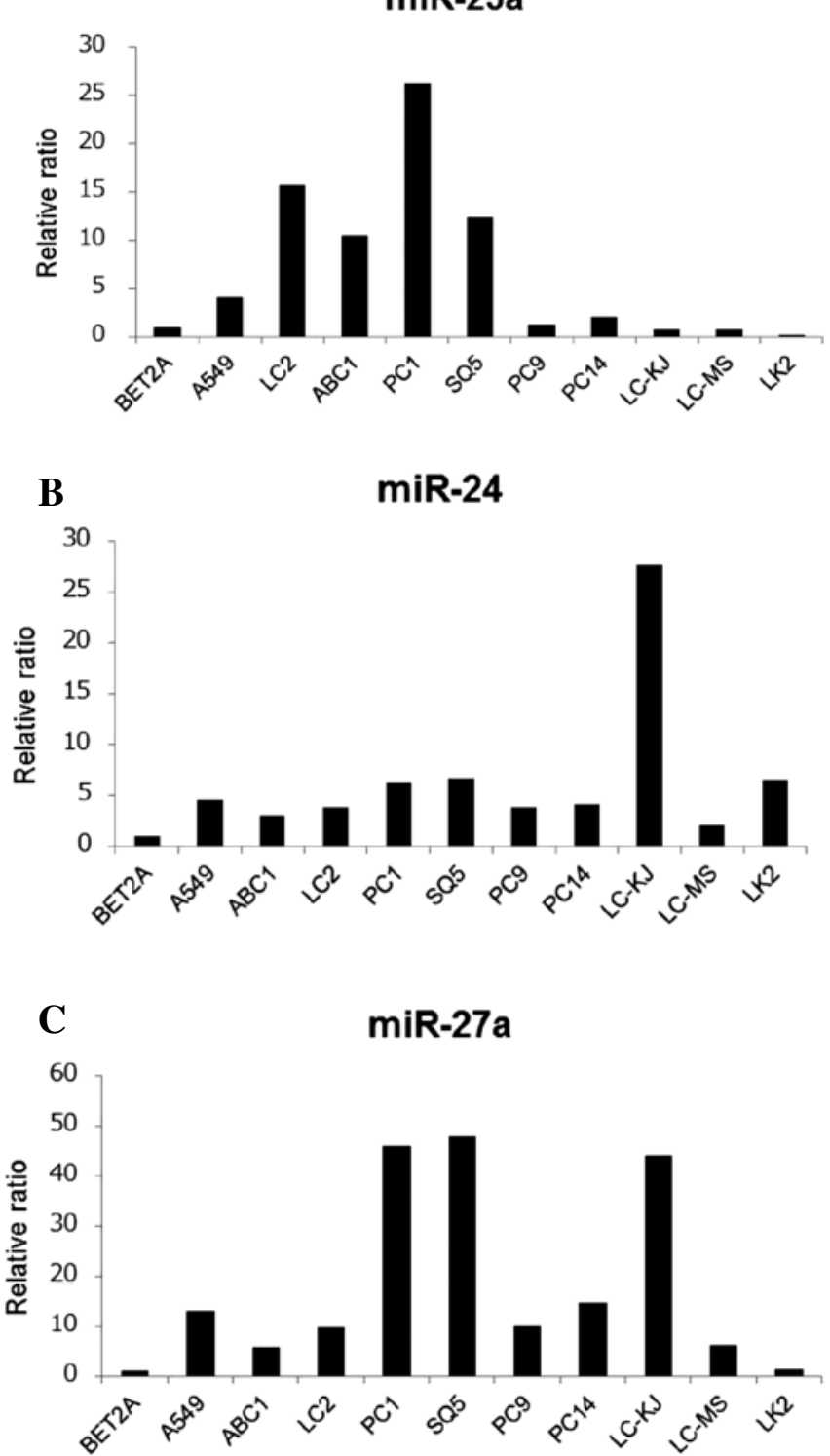

Figure 1. MiR-23a/24/27a expression in NSCLC cells. Expression levels of miR-23a/24/27a were analyzed in 10 NSCLC cells and BET2A cells by TaqMan ${ }^{\circledR}$ MicroRNA assay. MiRNA expression was quantified as $2^{-\Delta \Delta C t}$ value. Relative expressions of miR-23a/24/27 against BET2A cells are shown.

PC14, LC-KJ, LC-MS and LK2) (Fig. 2A). In contrast, no correlation was observed between miR-24 or miR-27a and Smad2/3.

It is well known that TGF- $\beta 1$ stimulates the EMT of A549 lung cancer cells (10). In this study, A549 cells which overexpressed miR-23a and Smad2/3 was treated with $5 \mathrm{ng} / \mathrm{ml}$ of TGF- $\beta 1$ for $48 \mathrm{~h}$. We observed that, while the parent A549 cells exhibited a classic epithelial morphology (Fig. 2B), after TGF- $\beta 1$ exposure they had a less uniform epithelial appearance (Fig. 2B). In contrast, PC14 cells with low expression of miR-23a and Smad2/3 retained their epithelial morphology after TGF- $\beta 1$ treatment (Fig. 2B). Using western blot analysis, we evaluated the expression levels of EMT markers in A549 and PC14 cells treated with TGF- $\beta 1$ in order to confirm the occurrence of EMT. A549 cells treated with TGF- $\beta 1$ displayed reduced E-cadherin expression and increased $\mathrm{N}$-cadherin expression when compared with A549 cells without TGF- $\beta 1$
A

A549 LC-2 ABC1 PC1 SQ5 PC9 PC14 LC-KJ LC-MS LK2

SMAD2

SMAD3

$\beta$-actin

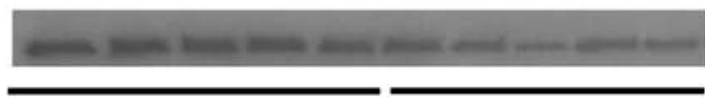

High miR-23a

Low miR-23a

B

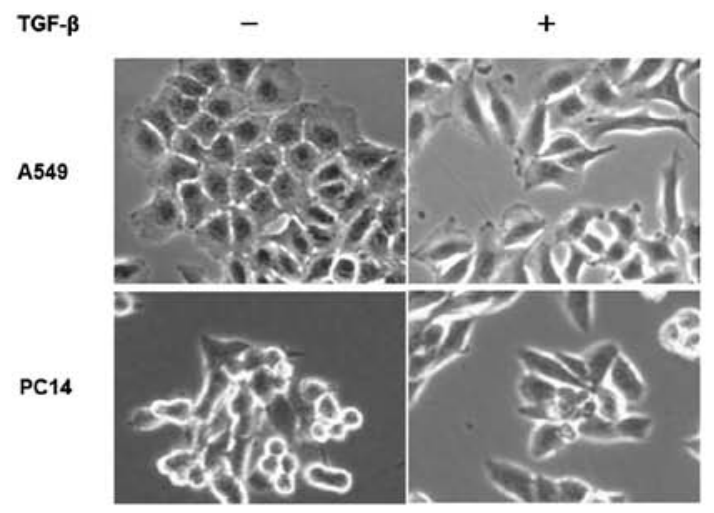

C

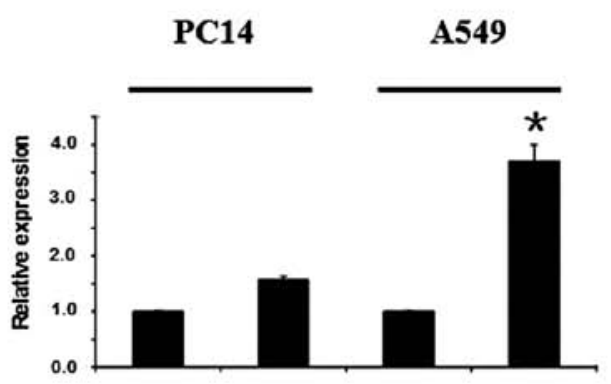

E-cadherin

N-cadherin

$\beta$-actin

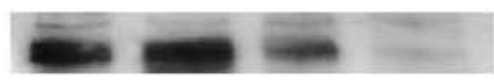

miR-23a
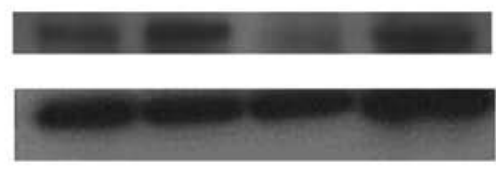

TGF- $\beta 1$

Figure 2. MiR-23a is directly induced by TGF- $\beta 1$ in a SMAD-dependent manner and involved in EMT of lung cancer cells. (A) Five cell lines (A549, LC-2, ABC1, PC1 and SQ5) showed high expression of both miR-23a and $\mathrm{Smad} 2 / 3$. In contrast, low expression of miR-23a and $\mathrm{Smad} 2 / 3$ were found in the other five cell lines (PC9, PC14, LC-KJ, LC-MS and LK2). (B) A549 and PC14 cells were treated with $5 \mathrm{ng} / \mathrm{ml}$ of TGF- $\beta 1$ for $48 \mathrm{~h}$. The cells were observed under a light microscope. A549 cells exhibited a classic epithelial morphology. In contrast, A549 cells after the TGF- $\beta 1$ exposure appeared to be less uniformly epithelial. (C) MiR-23a and protein expression of EMT markers in A549 and PC14 cells after TGF- $\beta 1$ stimulation. Compared with PC14 cells, high expression of miR-23a, lost E-cadherin expression and enhanced $\mathrm{N}$-cadherin expression were observed in A549 cells after TGF- $\beta 1$ stimulation.

stimulation (Fig. 2C). We also examined whether TGF- $\beta 1$ stimulated miR-23a expression in these two cells. MiR-23a expression level was significantly higher in A549 cells, which had shown the EMT phenomenon after the treatment of the respective parent cells with TGF- $\beta 1$ (Fig. $2 \mathrm{C}$ ). In contrast, miR-23a expression level in PC14 cells, which had not shown 

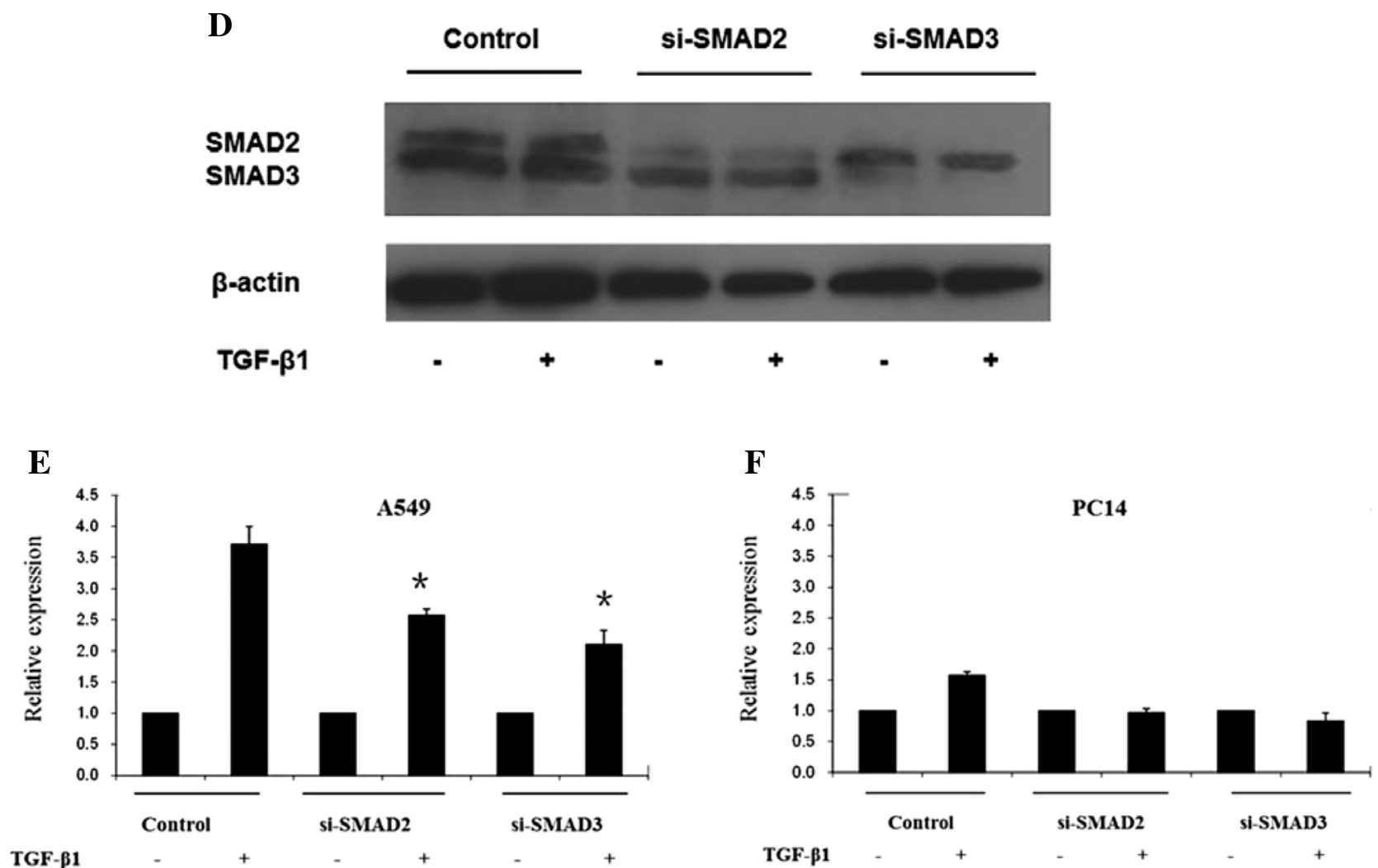

Figure 2. Continued. (D) Protein expression of Smad2/3 after treatment of siRNA in A549 cells. A549 cells were treated with control siRNA or siRNA of Smad $2 / 3$ with or without TGF- $\beta 1$ stimulation. Smad 2 or Smad 3 specific siRNA completely diminished Smad 2 or Smad 3 expression. (E) Expression of miR-23a after the transfection of Smad2/3 siRNA was evaluated. MiR-23a expression was significantly decreased after the treatment of Smad2 or Smad3 siRNAs with TGF- $\beta 1$ stimulation in A549 cells. (F) MiR-23a expression was unchanged after the transfection of Smad2 or Smad3 siRNAs with TGF- $\beta 1$ stimulation in PC14 cells. Data are mean \pm SD from 3 independent experiments. ${ }^{*}<<0.05$ when compared with the respective parent cells.

the EMT phenomenon, was unaffected by exposure to TGF- $\beta 1$ (Fig. 2C).

Next, we examined whether the Smad signal pathway directly regulated miR-23a expression. A549 and PC14 cells were treated with siRNAs of control or Smad2/3 (Fig. 2D). Knockdown of Smad2/3 significantly decreased TGF- $\beta 1$-induced miR-23a expression in A549 cells in which miR-23a was overexpressed (Fig. 2E). On the other hand, in PC14 cells with low miR-23a, miR23a expression was unaffected by treatment with Smad siRNAs (Fig. 2F). These results suggested that, in A549 lung cancer cells, miR-23a was directly regulated by TGF- $\beta 1 / \mathrm{Smad}$ pathway and contributed to the EMT phenomenon.

MiR-23a regulates TGF- $\beta 1$-induced EMT by targeting $E$-cadherin. Since miR-23a was significantly upregulated in A549 cells after the treatment with TGF- $\beta 1$ and mediated EMT, we proceeded to identify potential targets known to play a role in EMT by using the Target Scan database. Among the candidate miRNAs for the E-cadherin gene (CDH1), we found that the region of 3' UTR of the CDH1 gene may serve as a binding site for miR-23a based on the prediction of Target Scan database (Fig. 3A).

To examine whether the CDH1 was a target of miR-23a, we knocked down miR-23a in A549 cells by using a specific inhibitor. Control or specific miR-23a inhibitor was transfected into A549 cells for $24 \mathrm{~h}$, which were then treated with or without TGF- $\beta 1$ for a further $48 \mathrm{~h}$. We confirmed that miR-23a was effectively knocked down by miR-23a inhibitor in A549 cells (Fig. 3B). Using western blot analysis, we evaluated the expression of EMT markers after the treatment of miR-23a inhibitor to confirm the occurrence of EMT. After exposure to TGF- $\beta 1$, E-cadherin expression in A549 cells was greatly diminished, resulting in TGF- $\beta 1$-induced EMT (Fig. 3C). Interestingly, E-cadherin was still expressed in A549 cells transfected with miR-23a inhibitor after TGF- $\beta 1$ exposure (Fig. 3C). N-cadherin expression was also weak after miR-23a treatment followed by TGF- $\beta 1$ exposure (Fig. 3C). These findings demonstrated that miR-23a inhibition partially suppressed TGF- $\beta$-induced EMT phenomenon in A549 cells.

We transiently transfected A549 cells with the miR-23a precursor (Pre-miR-23a), and the control precursor miR (Pre miR-ctl). Mature miR-23a was remarkably induced by the miR-23a precursor in A549 cells between 24 to $72 \mathrm{~h}$ (Fig. 3D). After the treatment of precursor miR-23a,decreasedE-cadherin expression and increased levels of vimentin were observed in A549 cells at $48 \mathrm{~h}$ (Fig. 3E). Under a light microscope, overexpression of miR-23a enhanced the spindle integration, resulting in an additive effect with TGF- $\beta 1$-induced EMT in A549 cells (Fig. 3F). These results suggested that miR-23a may affect EMT by targeting E-cadherin in lung cancer cells.

MiR-23a stimulated EMT and induced resistance to gefitinib. Finally, to evaluate whether EMT leads to resistance against 
A H. sapiens CDH1 3' UTR

\section{$5 '$ '... AUCUCUUUUUAUUUAAAUGUGAA... \\ $3^{\prime}$ \\ | | | | | |}

hsa-miR-23a

B

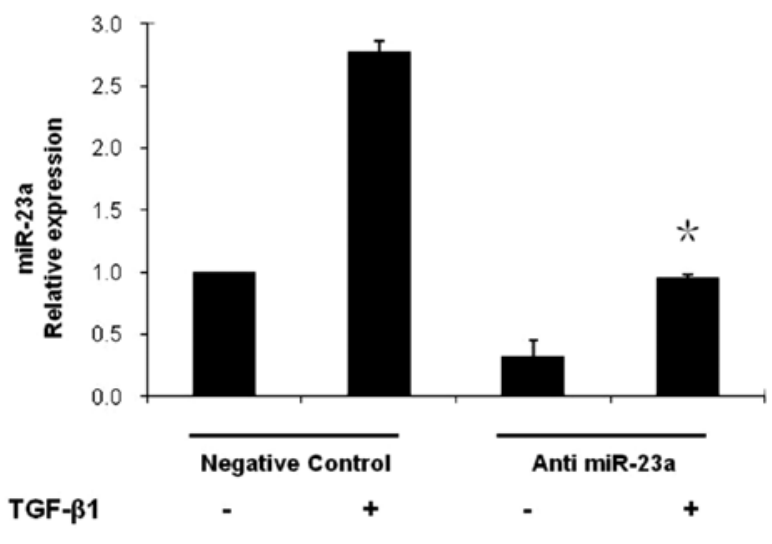

C

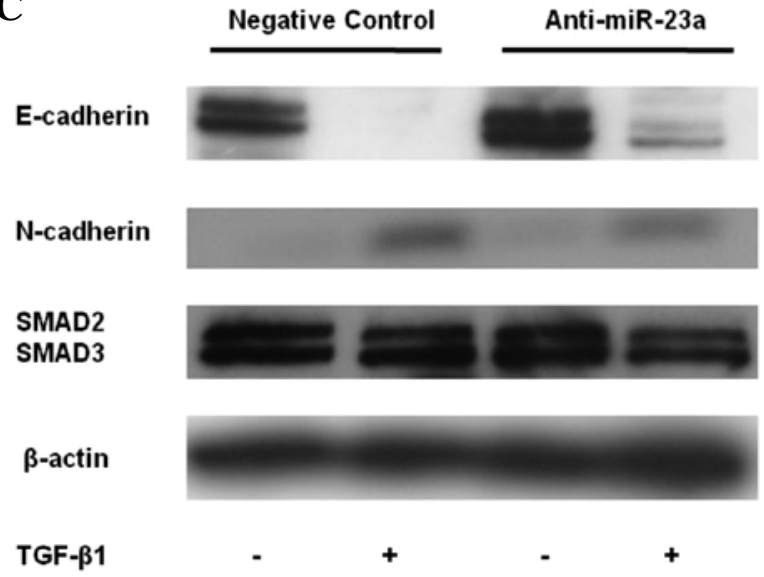

D

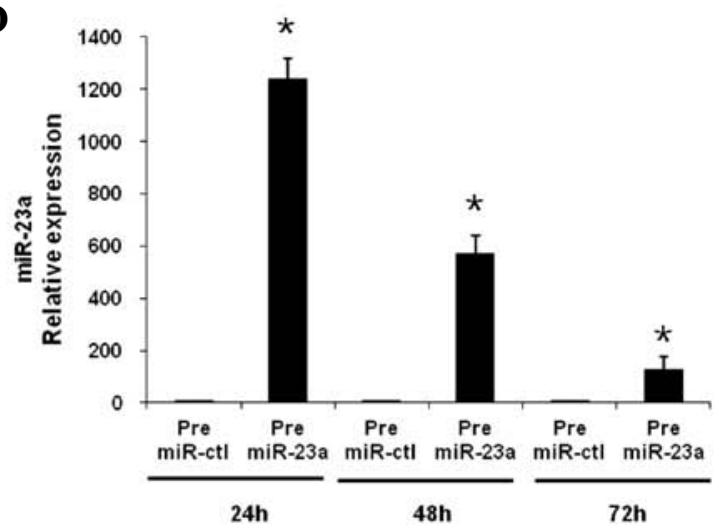

$\mathbf{E}$

\section{Pre-miR-ctl Pre-miR-23a}

E-cadherin

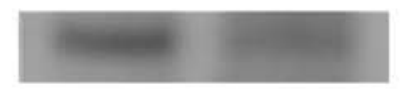

Vimentin

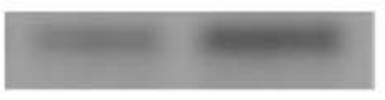

$\beta$-actin

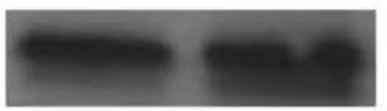

$\mathbf{F}$

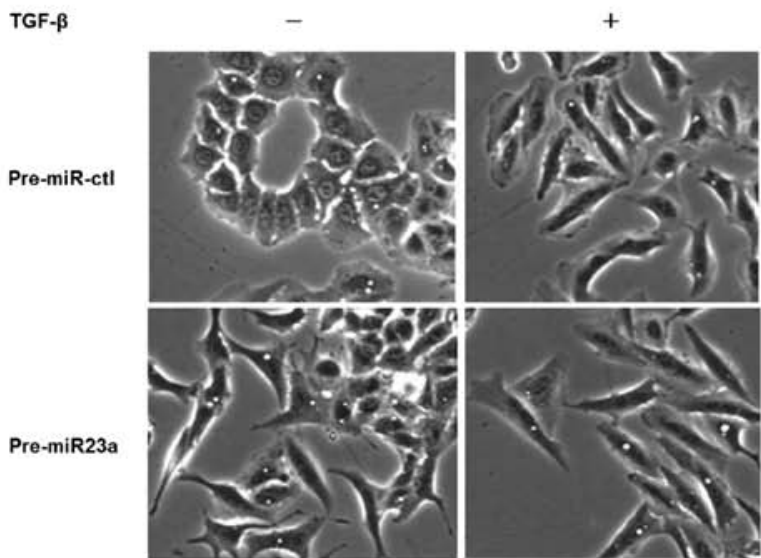

Figure 3. MiR-23a directly targets CDH1 and regulated TGF- 31 -induced EMT. (A) Predicted duplex formation between human CDH1 3' UTR and miR-23a. (B) Control or specific miR-23a inhibitor was transfected into A549 cells for $24 \mathrm{~h}$, which were then treated with or without TGF- $\beta 1$ for a further $48 \mathrm{~h}$. MiR-23a was significantly knocked down by miR-23a inhibitor in A549 cells. (C) EMT markers and SMAD2/3 expression were examined in A549 cells after the treatment with anti-miR-23a or control inhibitor. (D) Pre-miR-ctl or Pre-miR-23a was transfected into A549 cells for $72 \mathrm{~h}$. The histograms show the relative expression of mature miR-23a in A549 cells after treatment with Pre-miR-23a or Pre-miR-ctl, by qRT-PCR analysis. (E) At 48 h, E-cadherin expression was reduced in A549 cells treated with Pre-miR-23a. In contrast, at $48 \mathrm{~h}$, vimentin expression was increased in A549 cells transfected with Pre-miR-23a. (F) The cells were observed under a light microscope. A549 cells after the treatment of Pre-miR-23a appeared to have a spindle morphology.

the EGFR-TKI, gefitinib, we measured the response to gefitinib after the exposure of A549 cells to TGF- $\beta 1$ or Pre-miR-23a. Consistent with previous studies, A549 cells treated with TGF- $\beta 1$ were more resistant to gefitinib than were the parental cells (Fig. 4A). Interestingly, the resistance to gefitinib was also found in A549 cells after treatment with Pre-miR-23a only (Fig. 4A). The $\mathrm{IC}_{50}$ values of gefitinib with TGF- $\beta 1$ and gefitinib after treatment with Pre-miR-23a were 32 and 24, respectively, whereas that of gefitinib monotherapy was 6.8 (Fig. 4B). These findings indicate that induction of EMT affects cellular response to gefitinib and that miR-23a contributes to the resistance as well as TGF- $\beta 1$.

\section{Discussion}

TGF- $\beta 1$ has been recognized as a regulator of EMT in advancedstage human cancers, a phenomenon which promotes tumorigenesis, cancer progression and metastasis (10). Previous studies have demonstrated that EMT plays a key role in lung cancer progression $(15,28,29)$. In addition, it has been demonstrated that cancer progression might stimulate EMT in lung cancer, resulting in resistance to anticancer drugs (17-19). Therefore, the early detection of EMT development or attenuation of the EMT phenotype in lung cancer cells may be useful, particularly in helping to improve the clinical treatment of lung cancer. 

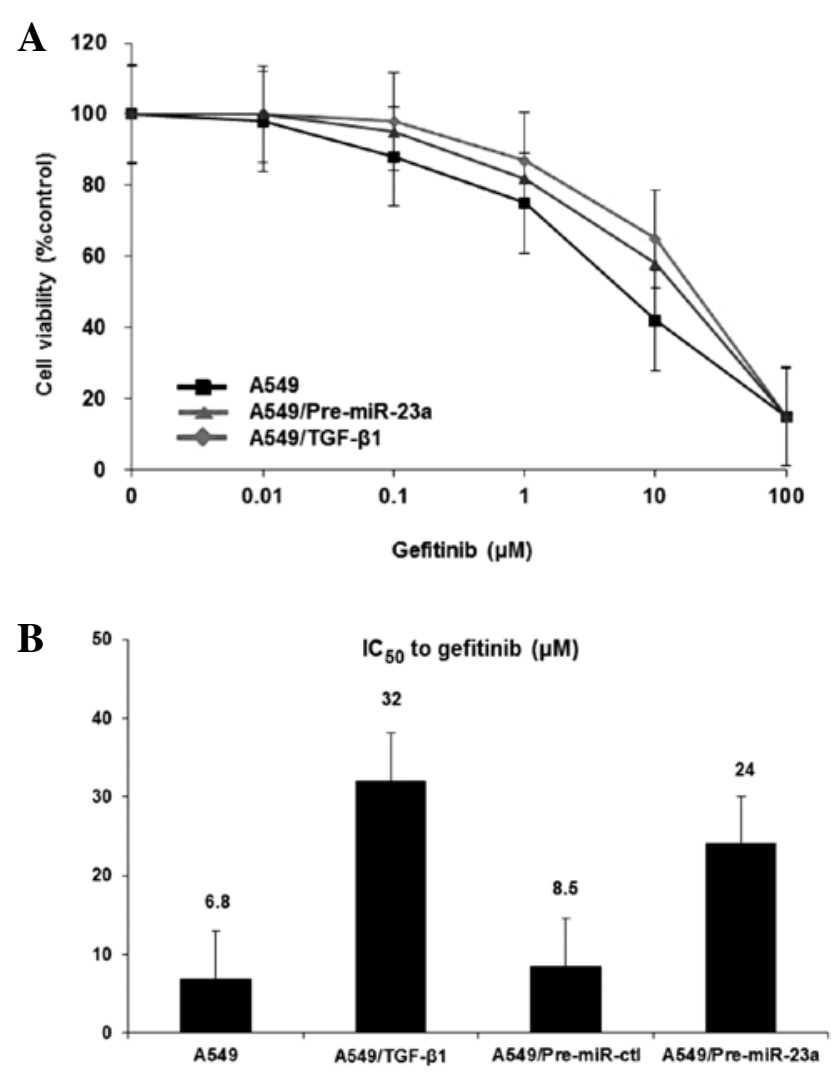

Figure 4. MiR-23a contributes to gefitinib drug resistance. (A) Gefitinib treatment with 5 ng TGF- $\beta 1$ or Pre-miR-23a or Pre-miR-ctl for $24 \mathrm{~h}$ was examined in A549 cells. Then, the cells were incubated in the various concentrations of gefitinib for $72 \mathrm{~h}$. (B) The $\mathrm{IC}_{50}$ values of gefitinib with TGF- $\beta 1$ and gefitinib after treatment with Pre-miR-ctl or Pre-miR-23a. Each result is expressed as cell viability in the treated samples compared with the untreated sample (100\%) for gefitinib therapy. Data are mean \pm SD from 3 independent experiments.

Recent reports showed that several miRNAs play a crucial role in the regulation of EMT of several cancers (30-34). The miR-200 family and miR-205 have been shown to contribute to EMT in cancer cells by the direct targeting of transcriptional repressors of E-cadherin, ZEB1 and ZEB2 (30-32). More specifically, in breast cancer cells, miR-155 has been shown to facilitate TGF- $\beta$-induced EMT by targeting RhoA (33). Finally, miR-9 activated by MYC/MYCN mediated E-cadherin downregulation resulting in the activation of $\beta$-catenin, and VEGF, and metastases in human cancers, including neuroblastomas and breast tumors (34). However, in lung cancer, the mechanism by which miRNA contributes to TGF- $\beta$-induced EMT is largely unknown.

MiR-23a/24/27a is a miRNA cluster located in chromosome 19p13.12 and can be induced by TGF- $\beta$ (26). This cluster functions as an oncogenic miRNA in several human cancers, and previous studies have reported that $\mathrm{miR}-23 \mathrm{a} / 24 / 27 \mathrm{a}$ was upregulated in human cancers $(26,35)$. Furthermore, miR-23a/24/27a functioned as a growth-promoting and antiapoptotic factor in HCC cells (26), while miR-23a was also shown to promote the growth of gastric adenocarcinoma cells and downregulate interleukin-6 receptor (35). In addition, c-myc suppression of miR-23a enhances mitochondrial glutamine metabolism and glutaminase expression (36). The cognate of glutamine is the major component that catabolizes glutamine to generate energy and lactate. Plenty of large amounts of glutamine are aggressively transported into cells to promote cancer cell proliferation and act as a source of carbon in the carbon cycle. Taken together, miR-23a/24/27a can be induced by TGF- $\beta$ and act as an oncogenic or tumor repressive miRNA in multiple human malignancies. However, the relation between miR-23a/24/27a and TGF- $\beta /$ Smad pathway remains unclear in lung cancer cells.

In this study, we found that expression of miR-23a was directly induced by the TGF- $\beta 1 /$ Smad pathway in A549 lung adenocarcinoma cells with the EMT phenomenon. In contrast, miR-24 and miR-27a belonging to the same cluster were induced in a Smad-independent manner in lung adenocarcinoma cells. We suggest that TGF- $\beta 1$ mainly regulates the expression of miR-23a in lung cancer cells. Furthermore, overexpression of miR-23a decreased E-cadherin expression and increased levels of vimentin, resulting in the EMT phenomenon in A549 lung cancer cells; silencing of miR-23a partially restored E-cadherin expression. This is the first report showing that miR-23a regulated TGF- $\beta 1$-induced EMT via E-cadherin suppression in lung cancer cells.

Molecular-targeted therapies have been recently developed for NSCLC treatment. NSCLC patients with EGFR gene mutations have shown a dramatic response to EGFR-TKIs such as gefitinib and erlotinib $(4,5)$. We have recently reported that first-line gefitinib for advanced NSCLC patients with EGFR mutations improved progression-free survival with acceptable toxicity $(5,37)$. However, it is recognized that, clinically, drug resistance eventually emerges and this limits the mean duration of response. Although mechanisms of acquired resistance, such as T790M secondary mutation and MET amplification, have recently been found, other mechanisms should be identified to widen the therapeutic strategy for NSCLC with EGFR mutations $(38,39)$. EMT has been reported to be correlated with an unfavorable prognosis for NSCLC patients (40). Some studies showed that the mesenchymal phenotype was more resistant to EGFR-TKI than the epithelial phenotype in NSCLC (17-19). Similarly, restoration of E-cadherin increased the sensitivity to EGFR-TKI in lung cancer cell lines (41). Consistent with previous findings, induction of EMT by TGF- $\beta 1$ in A549 cells led to the acquisition of resistance to gefitinib. Furthermore, overexpression of miR-23a induced EMT by suppressing E-cadherin expression and contributed to the reduced sensitivity to gefitinib in A549 cells. These findings demonstrated that suppression of EMT by miR-23a inhibition might overcome the resistance to EGFR-TKIs observed in NSCLC.

In conclusion, our study has provided evidence that miR-23a regulated TGF- $\beta$-induced EMT by suppression of E-cadherin and contributed to EGFR-TKI resistance in lung cancer cells. MiR-23a might be a potential prognostic marker and a new therapeutic target in NSCLC. Further studies should be performed to clarify the connection between miR-23a and TGF- $\beta$ /Smad signaling during the EMT process in NSCLC.

\section{Acknowledgements}

This study was supported in part by a Grant-in-Aid from the Ministry of Education, Culture, Sports, Science, and Technology (MEXT) of Japan, and MEXT-Supported Program for the Strategic Research Foundation at Private Universities (to M.S. and A.G.). 


\section{References}

1. Jemal A, Siegel R, Ward E, et al: Cancer Statistics, CA Cancer J Clin 59: 225-249, 2009.

2. Schiller JH, Harrington D, Belani CP, et al: Eastern Cooperative Oncology Group. Comparison of four chemotherapy regimens for advanced non-small-cell lung cancer. N Engl J Med 346: 92-98, 2002.

3. Ohe Y, Ohashi Y, Kubota K, et al: Randomized phase III study of cisplatin plus irinotecan versus carboplatin plus paclitaxel, cisplatin plus gemcitabine, and cisplatin plus vinorelbine for advanced non-small-cell lung cancer: Four-Arm Cooperative Study in Japan. Ann Oncol 18: 317-323, 2007.

4. Mok TS, Wu YL, Thongprasert S, et al: Gefitinib or carboplatinpaclitaxel in pulmonary adenocarcinoma. N Engl J Med 361: 947-957, 2009.

5. Maemondo M, Inoue A, Kobayashi K, et al: Gefitinib or chemotherapy for non-small-cell lung cancer with mutated EGFR. N Engl J Med 362: 2380-2388, 2010.

6. Beer DG, Kardia SL, Huang CC, et al: Gene-expression profiles predict survival of patients with lung adenocarcinoma. Nat Med 8: 816-824, 2002.

7. Potti A, Mukherjee S, Petersen R, et al: A genomic strategy to refine prognosis in early-stage non-small-cell lung cancer. $\mathrm{N}$ Engl J Med 355: 570-580, 2006.

8. Seike M, Yanaihara N, Bowman ED, et al: Use of a cytokine gene expression signature in lung adenocarcinoma and the surrounding tissue as a prognostic classifier. J Natl Cancer Inst 99: 1257-1269, 2007.

9. Thiery JP and Sleeman JP: Complex networks orchestrate epithelial-mesenchymal transitions. Nat Rev Mol Cell Biol 7: 131-142, 2006

10. Zavadil $\mathrm{J}$ and Bottinger EP: TGF- $\beta$ and epithelial-to-mesenchymal transitions. Oncogene 24: 5764-5774, 2005.

11. Derynck R and Zhang YE: Smad-dependent and Smadindependent pathways in TGF- $\beta$ family signalling. Nature 425 : 577-584, 2003.

12. Thiery JP: Epithelial-mesenchymal transitions in development and pathologies. Curr Opin Cell Biol 15: 740-746, 2003.

13. Thiery JP: Epithelial-mesenchymal transitions in tumour progression. Nat Rev Cancer 2: 442-454, 2002.

14. Huber MA, Kraut N and Beug H: Molecular requirements for epithelial-mesenchymal transition during tumor progression. Curr Opin Cell Biol 17: 548-558, 2005.

15. Singh A, Greninger P, Rhodes D, et al: A gene expression signature associated with 'K-Ras addiction' reveals regulators of EMT and tumor cell survival. Cancer Cell 15: 489-500, 2009.

16. Mizutani H, Okano T, Minegishi Y, et al: HSP27 modulates epithelial to mesenchymal transition of lung cancer cells in a Smad-independent manner. Oncol Lett 1: 1011-1016, 2010.

17. Yauch RL, Januario T, Eberhard DA, et al: Epithelial versus mesenchymal phenotype determines in vitro sensitivity and predicts clinical activity of erlotinib in lung cancer patients. Clin Cancer Res 11: 8686-8698, 2005.

18. Thomson S, Buck E, Petti F, et al: Epithelial to mesenchymal transition is a determinant of sensitivity of non-small-cell lung carcinoma cell lines and xenografts to epidermal growth factor receptor inhibition. Cancer Res 65: 9455-9462, 2005.

19. Rho JK, Choi YJ, Lee JK, et al: Epithelial to mesenchymal transition derived from repeated exposure to gefitinib determines the sensitivity to EGFR inhibitors in A549, a non-small cell lung cancer cell line. Lung Cancer 63: 219-226, 2009.

20. Johnson SM, Grosshans H, Shingara J, et al: RAS is regulated by the let-7 microRNA family. Cell 120: 635-647, 2005.

21. Lu J, Getz G, Miska EA, et al: MicroRNA expression profiles classify human cancers. Nature 435: 834-838, 2005.

22. Volinia S, Calin GA, Liu CG, et al: A microRNA expression signature of human solid tumors defines cancer gene targets. Proc Natl Acad Sci USA 103: 2257-2261, 2006.
23. Yanaihara N, Caplen N, Bowman E, et al: Unique microRNA molecular profiles in lung cancer diagnosis and prognosis. Cancer Cell 9: 189-198, 2006.

24. Hayashita Y, Osada H, Tatematsu Y, et al: A polycistronic microRNA cluster, miR-17-92, is overexpressed in human lung cancers and enhances cell proliferation. Cancer Res 65: 9628-9632, 2005

25. Seike M, Goto A, Okano T, et al: MiR-21 is an EGFR-regulated anti-apoptotic factor in lung cancer in never-smokers. Proc Natl Acad Sci USA 106: 12085-12090, 2009.

26. Huang S, He X, Ding J, et al: Upregulation of miR-23a approximately 27 a approximately 24 decreases transforming growth factor-beta-induced tumor-suppressive activities in human hepatocellular carcinoma cells. Int J Cancer 123: 972-978, 2008.

27. Bustin SA: Absolute quantification of mRNA using real-time reverse transcription polymerase chain reaction assays. J Mol Endocrinol 25: 169-193, 2000.

28. Saito RA, Watabe T, Horiguchi K, et al: Thyroid transcription factor-1 inhibits transforming growth factor-beta-mediated epithelial-to- mesenchymal transition in lung adenocarcinoma cells. Cancer Res 69: 2783-2791, 2009.

29. Soltermann A, Tischler V, Arbogast S, et al: Prognostic significance of epithelial-mesenchymal and mesenchymal-epithelial transition protein expression in non-small cell lung cancer. Clin Cancer Res 14: 7430-7437, 2008.

30. Gregory PA, Bert AG, Paterson EL, et al: The miR-200 family and miR-205 regulate epithelial to mesenchymal transition by targeting ZEB1 and SIP1. Nat Cell Biol 10: 593-601, 2008.

31. Korpal M, Lee ES, Hu G, et al: The miR-200 family inhibits epithelial-mesenchymal transition and cancer cell migration by direct targeting of E-cadherin transcriptional repressors ZEB1 and ZEB2. J Biol Chem 283: 14910-14914, 2008.

32. Tryndyak VP, Beland FA and Pogribny IP: E-cadherin transcriptional down-regulation by epigenetic and microRNA-200 family alterations is related to mesenchymal and drug-resistant phenotypes in human breast cancer cells. Int J Cancer 126: 2575-2583, 2010.

33. Kong W, Yang H, He L, et al: MicroRNA-155 is regulated by the transforming growth factor beta/Smad pathway and contributes to epithelial cell plasticity by targeting RhoA. Mol Cell Biol 28: 6773-6784, 2008.

34. Ma L, Young J, Prabhala H, et al: miR-9, a MYC/MYCN-activated microRNA, regulates E-cadherin and cancer metastasis. Nat Cell Biol 12: 247-256, 2010.

35. Zhu LH, Liu T, Tang H, et al: MicroRNA-23a promotes the growth of gastric adenocarcinoma cell line MGC803 and downregulates interleukin-6 receptor. FEBS J 277: 3726-3734, 2010.

36. Gao P, Tchernyshyov I, Chang TC, et al: c-Myc suppression of $\mathrm{miR}-23 \mathrm{a} / \mathrm{b}$ enhances mitochondrial glutaminase expression and glutamine metabolism. Nature 458: 762-765, 2009.

37. Inoue A, Kobayashi K, Usui K, et al: First-line gefitinib for patients with advanced non-small-cell lung cancer harboring epidermal growth factor receptor mutations without indication for chemotherapy. J Clin Oncol 27: 1394-1400, 2009.

38. Kobayashi S, Boggon TJ, Dayaram T, et al: EGFR mutation and resistance of non-small-cell lung cancer to gefitinib. N Engl J Med 352: 786-792, 2005.

39. Engelman JA, Zejnullahu K, Mitsudomi T, et al: MET amplification leads to gefitinib resistance in lung cancer by activating ERBB3 signaling. Science 316: 1039-1043, 2007.

40. Bremnes RM, Veve R, Gabrielson E, et al: High-throughput tissue microarray analysis used to evaluate biology and prognostic significance of the E-cadherin pathway in non-small-cell lung cancer. J Clin Oncol 20: 2417-2428, 2002.

41. Witta SE, Gemmill RM, Hirsch FR, et al: Restoring E-cadherin expression increases sensitivity to epidermal growth factor receptor inhibitors in lung cancer cell lines. Cancer Res 66: 944-945, 2006 